\title{
Activity-edge Centric Multi-label Classification for Mining Heterogeneous Information Networks
}

\author{
Yang Zhou \\ College of Computing \\ Georgia Institute of Technology \\ Atlanta, GA 30332 \\ yzhou@gatech.edu
}

\author{
Ling Liu \\ College of Computing \\ Georgia Institute of Technology \\ Atlanta, GA 30332 \\ lingliu@cc.gatech.edu
}

\begin{abstract}
Multi-label classification of heterogeneous information network$\mathrm{s}$ has received renewed attention in social network analysis. In this paper, we present an activity-edge centric multi-label classification framework for analyzing heterogeneous information networks with three unique features. First, we model a heterogeneous information network in terms of a collaboration graph and multiple associated activity graphs. We introduce a novel concept of vertex-edge homophily in terms of both vertex labels and edge labels and transform a general collaboration graph into an activitybased collaboration multigraph by augmenting its edges with class labels from each activity graph through activity-based edge classification. Second, we utilize the label vicinity to capture the pairwise vertex closeness based on the labeling on the activity-based collaboration multigraph. We incorporate both the structure affinity and the label vicinity into a unified classifier to speed up the classification convergence. Third, we design an iterative learning algorithm, AECLAss, to dynamically refine the classification result by continuously adjusting the weights on different activity-based edge classification schemes from multiple activity graphs, while constantly learning the contribution of the structure affinity and the label vicinity in the unified classifier. Extensive evaluation on real datasets demonstrates that AECLAss outperforms existing representative methods in terms of both effectiveness and efficiency.
\end{abstract}

\section{Categories and Subject Descriptors}

\section{H.2.8 [Database Applications]: Data Mining}

\section{Keywords}

Multi-label Classification; Heterogeneous Network; Activity-based Edge Classification; Collaboration Multigraph; Label Vicinity

\section{INTRODUCTION}

Multi-label classification has received increasing attention in both data mining and machine learning over the last decade [10-22]. In contrast to single-label classification, multi-label classification analysis adopts a more realistic view that entities in the real world are often associated with multiple class labels simultaneously. For example, most people in a social network belong to multiple social groups and participate in multiple types of activities with different

Permission to make digital or hard copies of all or part of this work for personal or classroom use is granted without fee provided that copies are not made or distributed for profit or commercial advantage and that copies bear this notice and the full citation on the first page. Copyrights for components of this work owned by others than ACM must be honored. Abstracting with credit is permitted. To copy otherwise, or republish, to post on servers or to redistribute to lists, requires prior specific permission and/or a fee. Request permissions from permissions@acm.org.

KDD'14, August 24-27, 2014, New York, NY, USA.

Copyright 2014 ACM 978-1-4503-2956-9/14/08 ...\$15.00.

http://dx.doi.org/10.1145/2623330.2623737. degrees of engagement. Most of web pages in the web graph may cover multiple topics at different intensities.

Existing multi-label classification efforts for networked data focus on designing effective and yet scalable algorithms [17-22]. Although previous studies differ from one another in the concrete approaches to mining the linkage structure, to the best of our knowledge, they all suffer from two weaknesses: (1) None of previous studies separate different types of activity graphs from the heterogeneous information networks and exploit the correlations among the set of class labels within each activity graph and across multiple activity graphs; and (2) None of previous works combine both the vertex-centric multi-label classification and the edge-centric multilabel classification to boost the effectiveness and efficiency.

In this paper we show that by utilizing activity-edge centric approach, we can incorporate the two missing dimensions to improve both the accuracy and the complexity of multi-label classification analysis. First, we argue that entities in the real world may involve themselves in multiple activity networks. These activity network$s$ may provide abundant information about heterogeneous entities and links, and how entities are linked in the context of each of activity networks. We aim to utilize these activity networks to find a natural and cheap way to identify the inter-dependencies among labels. Second, based on different activity networks, an entity can be tagged by a subset of $K$ labels with different class-membership distributions. We model the class-membership distribution for each of activity networks as multi-labeled edges. Third, we consider not only the labels of related vertices but also the possible labels of associated edges to further enhance the accuracy of multi-label classification. We integrate the vertex-centric labeling and edge-centric labeling into a unified classifier with different weights. An iterative method is proposed to learn the weights towards the classification objective.

This paper makes the following original contributions to multilabel classification for networked data.

- We model a heterogeneous information network in terms of a collaboration graph and multiple associated activity graphs, and cluster activity vertices in each activity graph into $K$ categories with the given $K$ class labels. Clustering each activity graph provides a natural way to capture the dependencies among activity categories within activity graphs.

- We introduce a novel concept of vertex-edge homophily in terms of both vertex labels and edge labels, and transform a general collaboration graph into an activity-based collaboration multigraph by augmenting its edges with class labels from each activity graph through activity-based edge classification.

- We utilize the structure affinity to capture the pairwise topological similarity of vertices and the label vicinity to capture the pairwise vertex closeness based on the labeling on the activitybased collaboration multigraph. We incorporate both the struc- 

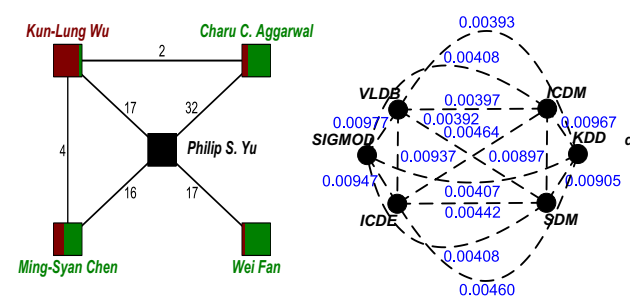

(a) Coauthor Graph (b) Conference Graph

Figure 1: An Illustrating Example from DBLP

ture affinity and the label vicinity into a unified classifier to speed up the classification convergence.

- We design an iterative learning algorithm, AEClass, to dynamically refine the classification result by continuously adjusting the weights on different activity-based edge classification schemes from multiple activity graphs, while constantly learning the contribution of the structure affinity and the label vicinity in the unified classifier. To make the classification process converge fast, a sophisticated nonlinear fractional programming problem with multiple weights is transformed to a straightforward parametric programming problem of a single variable.

- Empirical evaluation over real multi-label datasets demonstrates the competitiveness of AECLAss against state-of-the-art methods, in terms of both inference performance and time complexity.

\section{PROBLEM DEFINITION}

We address the problem of multi-label classification for networked data by employing our activity-edge centric multi-label classification algorithm. First, we model a heterogeneous information network in terms of two types of information networks: (1) a collaboration graph at the instance level, which is the target of multilabel classification, and (2) a collection of its associated activity graphs at the category level. For example, the DBLP bibliography dataset may consist of three types of vertices: authors, publication venues (e.g., conferences, journals), and title terms in the publications. An author can publish in multiple venues and his papers may contain multiple terms. If the target of multi-label classification is to infer author's labels, then we transform the DBLP dataset into a primary collaboration network for authors at the instance level and two associated activity networks (conference-similarity network and term-similarity network) at the category level. The collaboration network is defined based on both labeled and unlabeled instances with the given $K$ class labels, where each vertex represents one instance and each edge reflects the collaborative relationships between pairwise instances, e.g., the number of co-authored publications. Each of associated activity networks is constructed with all the associated activities as vertices. Similar activities are linked together with each edge value indicating the similarity between pairwise activities, such as product purchasing activity network, sport activity network or conference activity network. Given that each entity in the collaboration network may participate in multiple activities in each of activity networks, we cluster all activities in each activity networks into $K$ categories. Then we construct a collaboration multigraph by augmenting the original collaboration graph based on $N$ activity networks as follow: For each pair of vertices with an edge in the collaboration graph, if both have participated in at least one of $N$ activity networks, then we will add up to $K$ edges between this pair of vertices.

Figures 1 gives an illustrative example extracted from the DBLP dataset, consisting of three graphs: a collaboration graph of authors, a conference activity graph and a term activity graph. For ease of presentation, we only choose the co-authored papers published in three top DB conferences of SIGMOD, VLDB and ICDE, and three top DM conferences of $K D D, I C D M$ and $S D M$. In Figure

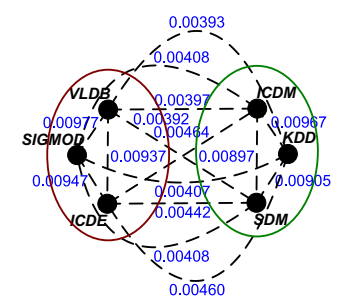

(a) Conference Clusters

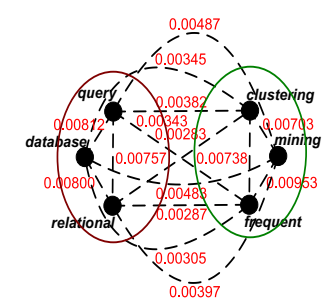

(b) Term Clusters
Figure 2: Activity Graph Partition

1 (a), ochre labels and green labels represent that authors are given predefined class labels of $D B$ and $D M$ respectively. In addition, ochre block or green block in each vertex rectangle represents the proportion of an author belonging to class $D B$ or $D M$. We want to use the label information of four labeled authors to learn the classmembership probabilities of Philip $S$. Yu over classes $D B$ and $D M$. In Figure 1 (b), blue numbers measure the similarity scores between pairwise conferences. We utilize a multi-typed soft clustering framework, NetClus [25], to cluster conferences and terms into 24 CS research areas [31] simultaneously. According to conference's clustering distribution over 24 categories and ranking score in each category, we calculate the similarities between conferences in the conference activity graph. Similarly, red numbers in Figure 1 (c) measure the similarity scores between terms. We then choose a category with the highest probability for each conference or each term as its primary category and put them into the corresponding primary categories. This operation actually produces a hard clustering result for each activity network. As shown in Figure 2, the conferences and terms in Figures 1 (b) and (c) are put into their individual primary categories respectively.

We formally define the above concepts as follows.

A collaboration graph is denoted as $C G=(V, F)$, where $V$ is the set of vertices representing the entities in $C G$, such as customers or authors, and $F$ is the set of edges denoting the collaborative relationships between members. We use $N_{C G}$ to represent the size of $V$, i.e., $N_{C G}=|V|$.

An activity graph is defined by $A G_{i}=\left(U_{i}, F_{i}\right)$, where $u \in U_{i}$ denotes an activity vertex in the $i^{\text {th }}$ associated activity network $A G_{i}$, and $f \in F_{i}$ is a weighted edge representing the similarity between two activity vertices, such as functional or manufacture similarity. We denote the size of each activity vertex set as $N_{A G_{i}}=\left|U_{i}\right|$. The vertex set $U_{i}$ is partitioned into $K$ disjoint primary categories, denoted by $U_{i p}(1 \leq p \leq K)$, such that $U_{i}=\bigcup_{p=1}^{K} U_{i p}$ and $U_{i p} \cap U_{i q}=$ $\phi$ for $\forall 1 \leq p, q \leq K, p \neq q$ and each activity category $U_{i p}$ is labeled with one of the $K$ class labels, $c_{p}$.

Given a collaboration graph $C G=(V, F)$ and its $N$ associated activity graphs $A G_{i}=\left(U_{i}, F_{i}\right)(1 \leq i \leq N)$, a collaboration multigraph denoted as $M G=(V, E)$, is an activity-edge augmented multigraph, where $V$ has the same definition in $C G$ and $E$ is the set of edges satisfying the following condition: for each edge $\left(v_{i}, v_{j}\right) \in F$ in $C G$, we create a set of parallel edges between the pair of vertices in $E$. Each set of edges has up to $K$ labeled edges and each edge corresponds to one activity category labeled by $c_{p}$ $(p \in\{1, \cdots, K\})$ in each of the $N$ activity graphs.

The problem of multi-label classification of multigraph is defined as follows: let $C=\left\{c_{1}, c_{2}, \cdots, c_{K}\right\}$ be a finite set of $K$ possible class labels. Given a collaboration multigraph $M G=(V, E)$ with a set of multi-label training instances $V_{l} \subset V$ initially labeled using the given $K$ class labels, and a set of multi-label testing instances $V_{u}=V-V_{l}$ unlabeled. For presentation brevity, we assume that the vertices in $V$ are ordered and the first $l$ vertices are labeled and the remaining vertices are unlabeled. Thus we have $V=\left\{v_{1}, \cdots, v_{l}, v_{l+1}, \cdots, v_{N_{C G}}\right\}$. Let an instance $v_{i} \in V$ be associated with a subset of labels in $C$,i.e., we use a binary vector 
$y_{i}=\left(y_{i}^{1}, y_{i}^{2}, \cdots, y_{i}^{K}\right) \in\{0,1\}^{K}$, in which $y_{i}^{j}=1$ iff the label $c_{j}$ is in the label set of $v_{i}$. We use $Y=\left\{y_{1}, \cdots, y_{l}, y_{l+1}, \cdots, y_{N_{C G}}\right\}$ to denote a possible labeling for the instance set $V . Y_{l}=\left\{y_{1}, \cdots, y_{l}\right\}$ indicates the observed multi-label set assigned to $V_{l}$ and $Y_{u}=Y-Y_{l}$ represents the multi-label set to be determined. The task of our activity-edge centric multi-label classification of multigraph is to use the label information of the training instances in $V_{l}$ to predict the label set $Y_{u}$ for the testing instances in $V_{u}$.

\section{THE AEClass APPROACH}

Compared to existing multi-label relational classifiers outlined in Section 1, AECLAss improves both the accuracy and the efficiency of multi-label classification by incorporating four mining strategies: (1) activity-based edge classification; (2) edge label dependency; (3) vertex label vicinity; and (4) weight learning. We first introduce the overall design of AECLAss. We then describe each part of AECLAss in detail in the next subsections.

- Activity-based edge classification, which consists of five tasks. (1) given a collaboration graph $C G$, choose $N$ suitable activity graphs $A G_{i}$ based on the specific context defined by the classification objective; (2) cluster all $A G_{i}$ s into $K$ activity categories; (3) construct an label dependency graph based on the clustering of each $A G_{i}$ to identify inter-dependencies among $K$ class labels; (4) based on $K$ categories of each $A G_{i}$, split and classify each unlabeled edge in $C G$ into at most $K$ labeled edges; and (5) transform $C G$ and all $A G_{i}$ s into a unified multigraph $M G$ by integrating $N$ edge classification schemes of $C G$ based on each $A G_{i}$ weighted by $\omega_{1}^{(1)}=\cdots=\omega_{N}^{(1)}=\frac{1}{N}$.

- Activity-edge centric vertex classification, which includes four tasks. (1) initialize a transition probability $\mathbf{T}_{j}^{(1)}$ of $M G$; (2) initialize a classification kernel $\mathbf{K}_{j}^{(1)}$; (3) infer the class-membership vector $\mathbf{X}_{j}^{(1)}$ on each class $c_{j}$; and (4) produce the class-membership vector $\mathbf{Y}_{j}^{(1)}$ by refining $\mathbf{X}_{j}^{(1)}$ with label dependency graphs.

- Iterative learning, which has four steps. (1) solve the parametric programming problem for classification objective to update $\alpha^{(t)}, \beta^{(t)}, \omega_{1}^{(t)}, \ldots, \omega_{N}^{(t)}\left(\alpha^{(t)}, \beta^{(t)}\right.$ are updated if $\left.t>2\right)$; (2) adjust the structure affinity $\mathbf{T}_{j}^{(t+1)}$ of $C G$ with $\omega_{1}^{(t)}, \ldots, \omega_{N}^{(t)} ;(3)$ update $\mathbf{K}_{j}^{(t+1)}$ by combining the structure affinity $\mathbf{T}_{j}^{(t+1)}$ and the label vicinity $\left(\mathbf{T}_{j}^{(t+1)} \mathbf{Y}^{(t)}\right)\left(\mathbf{T}_{j}^{(t+1)} \mathbf{Y}^{(t)}\right)^{T}$ weighted by $\alpha^{(t)}$ and $\beta^{(t)}\left(\alpha^{(t)}=\beta^{(t)}=\frac{1}{2}\right.$ if $t=2)$; and (4) do classification $\mathbf{X}_{j}^{(t+1)}=\mathbf{K}_{j}^{(t+1)} \mathbf{X}_{j}^{(t)}$ and enter the next round.

\subsection{Activity-based Edge Classification}

Existing classification models assume the existence of vertex homophily, namely, similar vertices in nature are connected to each other with social links. For example, Philip S. Yu and Wei Fan have many co-authored works published on $D M$ conferences, as shown in Figure 4 (a). However, the truth is not always like this. entities that are connected together may be similar in different ways with respect to a given set of $K$ class labels. As is known to all, Philip S. Yu and Ming-Syan Chen are experts on data mining, i.e., they both have more research publications in the area of data mining than in any other academic area such as database. However, as seen in Figure 4 (a), they have more co-authored papers published on $D B$ conferences. Thus the vertex homophily is insufficient to accurately infer the possible labels of an author. This motivates us to propose the concept of vertex-edge homophily, the principle that both links and their associated vertices should be similar and likely belong to the same classes, to further improve the accuracy of multi-label classification.

In order to capture the vertex-edge homophily in the multi-label classification, we first perform activity-based edge classification.

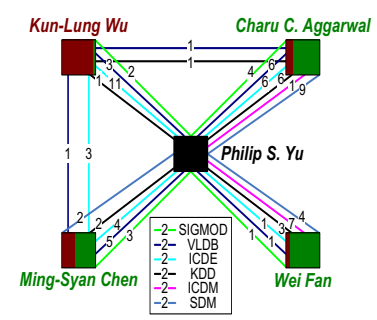

(a) Conference-based Splitting

\section{Figure 3: Edge Splitting}

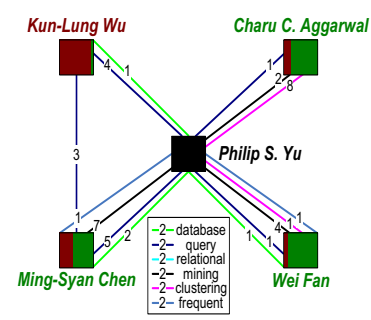

(b) Term-based Splitting
For each activity graph and the original collaboration graph $C G$, we first construct an activity-edge augmented collaboration graph $C G_{i}$ by examining each edge and the pair of connected entities in $C G$ and splitting each edge into a set of parallel edges based on each activity in $A G_{i}$ that this pair of entities have in common. The size of each set of parallel edges is at most $N_{A G_{i}}$, i.e., the number of activity vertices in $A G_{i}$. Figures 3 (a) and (b) present the activity-edge augmented collaboration graphs of Figure 1 (a) based on conference activities in Figure 1 (b) and term activities in Figure (c) respectively. Each edge in Figure 1 (a) is divided into multiple edges in terms of the common conference venues or the common title terms in co-authored publications between the pair of co-authors.

However, such activity-based edge augmentation may lead to substantial increase in size of activity-edge augmented collaboration graphs. We address this issue by introducing activity-based edge augmentation with edge classification to efficiently improve the scalability of classification. Concretely, we utilize the clustering result by NetClus, i.e., the probability distribution of each activity over $K$ categories, to infer the class labels of parallel edges in each $C G_{i}$ over the $K$ categories.

Given the probability of the $m^{\text {th }}$ activity in $A G_{i}$ belonging to cluster (class) $c_{j}$ produced by NetClus, denoted by $P\left(L_{m}=c_{j} \mid A G_{i}\right)$, we can compute the class-membership probability of edge $\left(v_{p}, v_{q}\right) \in E$ belonging to class $c_{j}$ based on $A G_{i}$, denoted by $P\left(L_{p q}=c_{j} \mid A G_{i}\right)$.

$$
P\left(L_{p q}=c_{j} \mid A G_{i}\right)=\frac{1}{W(p, q)} \sum_{m=1}^{N_{A G_{i}}} W_{m}^{i}(p, q) P\left(L_{m}=c_{j} \mid A G_{i}\right)
$$

where $W(p, q)$ represents the value on edge $\left(v_{p}, v_{q}\right) \in E$ in $C G$, and $W_{m}^{i}(p, q)$ denotes the value on the $m^{\text {th }}$ edge between $v_{p}$ and $v_{q}$ in $C G_{i}$, which is based on the $m^{\text {th }}$ activity in $A G_{i}$. If there does not exist such an edge between $v_{p}$ and $v_{q}$, then $W_{m}^{i}(p, q)$ is equal to 0 .

After generating the class-membership distribution of each edge in $C G_{i}$, we reduce $C G_{i}$ to an activity-edge augmented collaboration graph $\overline{C G_{i}}$ with classified edges by grouping at most $N_{A G_{i}}$ parallel edges between any pair of vertices in $C G_{i}$ into at most $K$ parallel edges in $\overline{C G_{i}}$.

$$
\bar{W}_{j}^{i}(p, q)=W(p, q) P\left(L_{p q}=c_{j} \mid A G_{i}\right)
$$

where $\bar{W}_{j}^{i}(p, q)$ represents the value on the edge with label $c_{j}$ between $v_{p}$ and $v_{q}$ in $\overline{C G_{i}}$. For ease of presentation, assuming that SIGMOD, VLDB, ICDE, database, query and relational only belong to class $D B$ with the probability of 1, and $K D D, I C D M, S D M$, mining, clustering and frequent just belong to class $D M$ with the probability of 1, two $\overline{C G_{i}}$ s in Figure 4 present the edge-classification results of two $C G_{i}$ s in Figures 3 respectively.

\subsection{Activity-edge Centric Vertex Classification}

As $N$ edge classification schemes of $C G$, i.e., $\overline{C G_{i}} \mathrm{~s}(1 \leq i \leq N)$, may have different degree of contributions to vertex classification, we propose to integrate $N$ edge classification schemes into a unified collaboration multigraph with different weighting factors $\omega_{1}^{(t)}, \cdots$, $\omega_{N}^{(t)}$ through dynamic weight tuning mechanism. Thus the unified weight value on the edge with label $c_{j}$ between $v_{p}$ and $v_{q}$ in $M G$ at the $t^{\text {th }}$ iteration, denoted by $\mathbf{W}_{j}^{(t)}(p, q)$, can be computed as follow. 


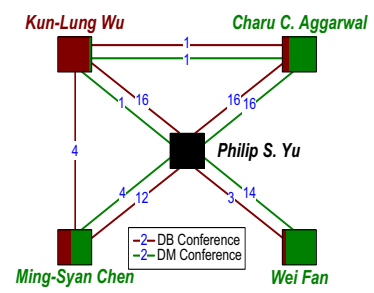

(a) Conference-based Classification

Figure 4: Edge Classification

$\mathbf{W}_{j}^{(t)}(p, q)=\sum_{i=1}^{N} \omega_{i}^{(t)} \bar{W}_{j}^{i}(p, q)=\sum_{i=1}^{N} \omega_{i}^{(t)} \sum_{m=1}^{N_{A G_{i}}} W_{m}^{i}(p, q) P\left(L_{m}=c_{j} \mid A G_{i}\right), 1 \leq j \leq K$

subject to $\sum_{i=1}^{N} \omega_{i}^{(t)}=1, \omega_{i}^{(t)} \geqslant 0, i=1, \cdots, N$.

Note that $\mathbf{W}_{j}^{(t)}(p, q)$ keeps changing with $\omega_{1}^{(t)}, \cdots, \omega_{N}^{(t)}$ through dynamic weight learning. We set the initial $\mathbf{W}_{j}^{(1)}(p, q)$ with equal weighting factors of $\omega_{1}^{(1)}, \cdots, \omega_{N}^{(1)}=\frac{1}{N}$.

Figure 5 (a) shows the unified multigraph for our running example in Figure 1 by combining the links with the same labels between the same vertex pair from two activity-based edge classification schemes in Figure 4 with equal weighting factor of 0.5.

With the unified multigraph $M G$, we below describe the activityedge centric vertex classification, which integrates the activity-edge labels with the vertex labels among structurally relevant instances through transition probability on collaboration multigraph.

Definition 1. [Transition Probability on Collaboration Multigraph] Let $M G=(V, E)$ be a collaboration multigraph where $V$ is the set of entity vertices and $E$ is the set of parallel edges denoting the collaborative relationships on different classes between entities of $M G$. The transition probability on $M G$ at the $t^{\text {th }}$ iteration can be defined by normalizing the edge values as follows.

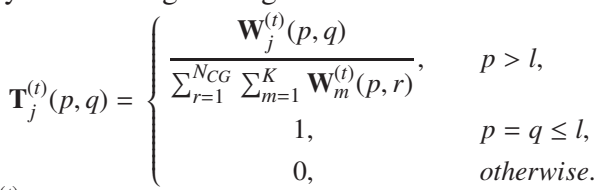

where $\mathbf{T}_{j}^{(t)}(p, q)$ represents the transition probability on the edge with label $c_{j}$ between $v_{p}$ and $v_{q}$ in $M G$. Here, we assume that $Y_{l}$, i.e., the labels of the vertices in $V_{l}$, are fixed during the classification process. Figure 5 (b) presents the transition probabilities of parallel edges from Philip S. Yu to other authors based on the collaboration multigraph in Figure 5 (a).

We express the above transition probability in a matrix form.

$$
\mathbf{T}_{j}^{(t)}=\left(\mathbf{D}^{(t)}\right)^{-1} \mathbf{W}_{j}^{(t)}
$$

where $\mathbf{D}^{(t)}$ is a diagonal matrix $\mathbf{D}^{(t)}=\operatorname{diag}\left(1, \cdots, 1, d_{l+1}, \cdots, d_{N_{C G}}\right)$, $1, \cdots, 1$ specifies $l$ ones, and $d_{p}=\sum_{r=1}^{N_{C G}} \sum_{m=1}^{K} \mathbf{W}_{m}^{(t)}(p, r)(l+1 \leq p \leq$ $\left.N_{C G}\right) . \mathbf{T}_{j}^{(t)}$ determines the transition probability on those edges with the class label of $c_{j}$ in $M G$.

Instead of decomposing the multi-label classification problem into a set of binary classification problems, we construct a unified multi-label classifier by using a single normalizing factor $\mathbf{D}^{(t)}$ to normalize parallel edges with different class labels. The original transition operation is actually divided into two steps: (1) choose those edges with the objective class label in terms of classification objective; and (2) select an edge with the largest value from the above edges to jump.

Now we define the initial unified classification kernel $\mathbf{K}_{j}^{(1)}$, which only utilizes the structure information of $M G$, i.e., those edges with label $c_{j}$, due to the lack of label vicinity at initialization.

$$
\mathbf{K}_{j}^{(1)}=\mathbf{T}_{j}^{(1)}
$$

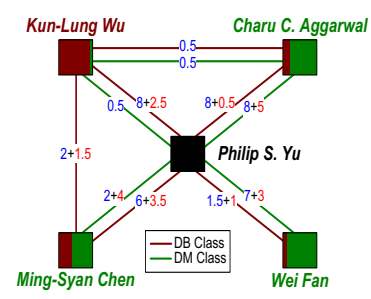

(a) Coauthor Multigraph

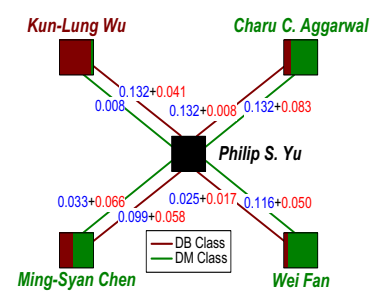

(b) Transition Probability
Figure 5: Multigraph Representation

Since we have ordered the vertices in $V$ such that the labeled nodes $V_{l}$ are indexed before the unlabeled nodes $V_{u}$, we rewrite the unified classification kernel $\mathbf{K}_{j}^{(1)}$ as a block matrix.

$$
\mathbf{K}_{j}^{(1)}=\left[\begin{array}{ll}
\mathbf{K}_{j l l}^{(1)} & \mathbf{K}_{j l u}^{(1)} \\
\mathbf{K}_{j u l}^{(1)} & \mathbf{K}_{j u u}^{(1)}
\end{array}\right]=\left[\begin{array}{cc}
\mathbf{I} & \mathbf{O} \\
\mathbf{K}_{j u l}^{(1)} & \mathbf{K}_{j u u}^{(1)}
\end{array}\right]
$$

where $\mathbf{K}_{j l l}^{(1)}$ is an $l \times l$ identity matrix representing the transition probability among labeled vertices, we set the $l \times\left(N_{C G}-l\right)$ block matrix $\mathbf{K}_{j l u}^{(1)}$ to be zero matrix since the labels on the vertices in $V_{l}$ are fixed, the $\left(N_{C G}-l\right) \times l$ matrix $\mathbf{K}_{j u l}^{(1)}$ specifies the transition probability from unlabeled vertices to labeled vertices, and $\mathbf{K}_{j u u}^{(1)}$ is an $\left(N_{C G}-l\right) \times\left(N_{C G}-l\right)$ matrix denoting the transition probability among unlabeled vertices.

Suppose that the class-membership matrix is denoted by $\mathbf{X}=$ $\left[\mathbf{X}_{1}, \mathbf{X}_{2}, \cdots, \mathbf{X}_{K}\right] \in \mathbb{R}^{N_{C G} \times K}$, for each class-membership vector $\mathbf{X}_{j}(1 \leq$ $j \leq K$ ) based on class $c_{j}$, we use its individual classification kernel $\mathbf{K}_{j}^{(t)}$ to iteratively infer the probabilities of vertices on class $c_{j}$.

$$
\mathbf{X}_{j}^{(t)}=\mathbf{K}_{j}^{(t)} \mathbf{X}_{j}^{(t-1)}
$$

Let $\mathbf{X}_{j}=\left[\mathbf{X}_{j l} ; \mathbf{X}_{j u}\right]$ be the class-membership vector, where $\mathbf{X}_{j l}$ indicates the probabilities of the labeled vertices in $V_{l}$ belonging to class $c_{j}$, and $\mathbf{X}_{j u}$ represents the probabilities of the unlabeled vertices in $V_{u}$ belonging to class $c_{j}$. Due to the labels on the vertices in $V_{l}$ are fixed, Eq. (8) is equivalent to the following formula.

$$
\mathbf{X}_{j u}^{(t)}=\mathbf{K}_{j u l}^{(t)} \mathbf{X}_{j l}^{(t-1)}+\mathbf{K}_{j u u}^{(t)} \mathbf{X}_{j u}^{(t-1)}
$$

After the $t^{\text {th }}$ iteration, the class-membership matrix is updated as follow.

$$
\mathbf{X}^{(t)}=\left[\mathbf{X}_{1}^{(t)}, \mathbf{X}_{2}^{(t)}, \cdots, \mathbf{X}_{K}^{(t)}\right]=\left[\begin{array}{llll}
\mathbf{X}_{1 l} & \mathbf{X}_{2 l} & \cdots & \mathbf{X}_{K l} \\
\mathbf{X}_{1 u}^{(t)} & \mathbf{X}_{2 u}^{(t)} & \cdots & \mathbf{X}_{K u}^{(t)}
\end{array}\right]
$$

Compared to existing multi-label relational classifiers, we argue that AEClass based on the activity-edge augmented collaboration multigraph can significantly improve the performance of multi-label classification: (1) accuracy improvement. Based on the vertex-edge homophily, we classify each edge in $C G$ into at most $K$ parallel edges in $M G$. During the classification process, AEClass only picks up those vertices and links with the same label as the current objective class $c_{j}$, i.e., $\mathbf{K}_{j}^{(t)}$ and $\mathbf{X}_{j}^{(t-1)}$, to execute the inference. For example, given the class-membership probabilities of Ming-Syan Chen on classes $D B$ and $D M$ in Figure 5 (a), we want to infer the class-membership probabilities of Kun-Lung Wu on $D B$ and $D M$. AEClass will produce a positive probability on $D B$ and a zero probability on $D M$ since there exists no edge with label $D M$ between these two authors. In contrast, existing classifiers will output a higher probability on $D M$ than on $D B$ for any positive edge value between two authors in the original $C G$ in Figure 1 (a). In fact, Kun-Lung $W u$ is known as a database researcher without any data mining publications. (2) efficiency improvement. Based on the vertex homophily, no matter which class the current objective is, existing classifiers need to check each neighbor of a vertex and summarize the labels of all neighbors. In comparison, AEClass performs the similar summary at lower cost. When we classify an edge in $C G$ into $m$ parallel edges in $M G, m$ is often much smaller than the number of $K$ class labels. Suppose that the current objective 


\section{DB $--0.00419-0.0$}

(a) Conference Dependency

Figure 6: Edge Label Dependency by Category Similarity

class is $c_{j}$, for a neighbor of a vertex, there may not exist an edge with label $c_{j}$ between the vertex and this neighbor. Thus, the number of neighbors of a vertex with edge label $c_{j}$ can be much smaller than the number of its neighbors, thus reducing the amount of unnecessary computations. Concretely, by utilizing the vertex-edge homophily, AEClass only needs to consider those links with label $c_{j}$ and associated neighbors and further stops label propagation to the circle of those irrelevant neighbors (without link with label $c_{j}$ ) in the next iterations. For the above example, we can safely ignore the operation of inferring the probability of Kun-Lung $W u$ on $D$ $M$ since there exists no edge with label $D M$ between two authors. More importantly, AEClass prevents the probability of Ming-Syan Chen on DM from being diffused to both Kun-Lung Wu and the neighbor-based circle of Kun-Lung Wu.

\subsection{Improvement by Edge Label Dependency}

We argue that the underlying correlations among different activity categories can have significant impact on the performance of multi-label classification. Based on activity graph partition, we first define the edge label similarity to capture the inter-dependencies among $K$ activity categories within each of $N$ activity graphs.

Definition 2. [Edge Label Similarity] Let $A G_{i}=\left(U_{i}, F_{i}\right)$ be the $i^{\text {th }}$ activity graph $(1 \leq i \leq N), S_{i}\left(u_{m}, u_{n}\right)$ be the similarity score between two activities $u_{m}, u_{n} \in U_{i}$ in $A G_{i}$, and $U_{i p}$ and $U_{i q}$ be two categories of $U_{i}$ with class labels of $c_{p}, c_{q} \in C$ respectively. The activity category similarity between $c_{p}$ and $c_{q}$ with respect to $A G_{i}$ is also referred to as the edge label dependency between two edge labels $c_{p}$ and $c_{q}$, and is defined as follow.

$$
S_{i}\left(c_{p}, c_{q}\right)=\left\{\begin{array}{cc}
\sum_{u_{m} \in U_{i p}, u_{n} \in U_{i q}} \frac{S_{i}\left(u_{m}, u_{n}\right)}{\left|U_{i p}\right| \times\left|U_{i q}\right|}, & p \neq q, \\
1, & p=q .
\end{array}\right.
$$

Figure 6 shows two edge label dependency graph by activity category similarity based on two class labels $D B$ and $D M$ with respect to the conference graph and the term graph in Figure 2 respectively.

We thus incorporate them into our AEClass framework to adjust the class-membership matrix $\mathbf{X}^{(t)}$. The adjusted class-membership vector on class $c_{j}$, denoted by $\mathbf{Y}_{j u}^{(t)}$, can be defined by integrating class-membership vectors on other classes in terms of the similarity scores between class $c_{j}$ and other classes.

$$
\mathbf{Y}_{j u}^{(t)}=\sum_{m=1}^{K} \sum_{i=1}^{N} \omega_{i}^{(t)} S_{i}\left(c_{j}, c_{m}\right) \mathbf{X}_{m u}^{(t)}, 1 \leq j \leq K
$$

where the weight $\omega_{i}^{(t)}$ for $A G_{i}$ is the same as in Eq. (3). The adjusted class-membership matrix is thus defined as follow.

$$
\mathbf{Y}^{(t)}=\left[\mathbf{Y}_{1}^{(t)}, \mathbf{Y}_{2}^{(t)}, \cdots, \mathbf{Y}_{K}^{(t)}\right]=\left[\begin{array}{llll}
\mathbf{X}_{1 l} & \mathbf{X}_{2 l} & \cdots & \mathbf{X}_{K l} \\
\mathbf{Y}_{1 u}^{(t)} & \mathbf{Y}_{2 u}^{(t)} & \cdots & \mathbf{Y}_{K u}^{(t)}
\end{array}\right]
$$

\subsection{Refinement by Vertex Label Vicinity}

One disadvantage of conventional iterative classifiers is that they often need lots of iterations to converge to a stationary distribution and the repeated label propagation causes a non-trivial computational cost. Wang et al. [22] proposed a dynamic label propagation (DLP) model by fusing both data features and data labels to improve the effectiveness on multi-class/multi-label classification. However, the DLP model failed to quantify the weighted contributions from data features and data labels such that it often can not work well on real classification tasks. We model the label vicinity to capture the pairwise vertex closeness based on the labeling on the activity-based collaboration multigraph by following the similar idea. To improve both effectiveness and efficiency of classification, we design an iterative learning method to dynamically refine the classification results by continuously quantifying and adjusting the weights on the structure affinity and on the label vicinity towards the classification objective.

Based on the transition probability $\mathbf{T}_{j}^{(t)}$ on $C G$, we define a diffusion process to map the multigraph space into an $N_{C G}$-dimensional space $\mathbb{R}^{N_{C G}}$, where each element $\phi_{j}^{(t)}(i) \in \mathbb{R}^{N_{C G}}$ represents the transition probabilities on the edges with label $c_{j}$ from vertex $v_{i}$ to the other vertices and $P\left(\phi_{j}^{(t)}(i)\right)=\mathcal{N}\left(\phi_{j}^{(t)}(i) \mid \mathbf{T}_{j}^{(t)}\right)$. On the other hand, based on a heuristics rule: two instance vertices with highly similar class-membership distributions are likely to be highly similar to each other in the input multigraph space, $\mathbf{Y}^{(t)}\left(\mathbf{Y}^{(t)}\right)^{T}$ can be viewed as the similarity between vertices based on the class-membership distribution. Similarly, we map this label-based similarity space into an $N_{C G}$-dimensional space $\mathbb{S}^{N_{C G}}$, where each entry $\varphi^{(t)}(i) \in \mathbb{S}^{N_{C G}}$ specifies the label-based similarity between vertex $v_{i}$ and the other vertices and $P\left(\varphi^{(t)}(i)\right)=\mathcal{N}\left(\varphi^{(t)}(i) \mid \mathbf{Y}^{(t)}\left(\mathbf{Y}^{(t)}\right)^{T}\right)$. We then define a linear projection operation based on $\mathbf{T}_{j}^{(t+1)}$.

$$
\phi_{j}^{(t+1)}=\mathbf{T}_{j}^{(t+1)} \varphi^{(t)}
$$

where $\phi_{j}^{(t+1)}=\left[\phi_{j}^{(t+1)}(1) ; \phi_{j}^{(t+1)}(2) ; \cdots ; \phi_{j}^{(t+1)}\left(N_{C G}\right)\right]$ and $\varphi^{(t)}=\left[\varphi^{(t)}(1)\right.$; $\left.\varphi^{(t)}(2) ; \cdots ; \varphi^{(t)}\left(N_{C G}\right)\right]$.

With the linear projection, we generate the following formula.

$$
P\left(\phi_{j}^{(t+1)} \mid \varphi^{(t)}\right)=\mathcal{N}\left(\phi_{j}^{(t+1)} \mid \mathbf{T}_{j}^{(t+1)} \varphi^{(t)}\right)
$$

The corresponding marginal distribution is given below.

$$
\begin{aligned}
P\left(\phi_{j}^{(t+1)}\right) & =\int \mathcal{N}\left(\varphi^{(t)} \mid \mathbf{Y}^{(t)}\left(\mathbf{Y}^{(t)}\right)^{T}\right) \mathcal{N}\left(\phi_{j}^{(t+1)} \mid \mathbf{T}_{j}^{(t+1)} \varphi^{(t)}\right) d \varphi^{(t)} \\
& =\mathcal{N}\left(\phi_{j}^{(t+1)} \mid \mathbf{T}_{j}^{(t+1)} \mathbf{Y}^{(t)}\left(\mathbf{Y}^{(t)}\right)^{T}\left(\mathbf{T}_{j}^{(t+1)}\right)^{T}\right)
\end{aligned}
$$

Since directly combining $\varphi^{(t)}\left(\varphi^{(t)}\right)^{T}$ into the unified classification kernel $\mathbf{K}_{j}^{(t+1)}$ may lead to a degeneration at the beginning of classification if the learned label information of vertices in $V_{u}$ is not enough to infer the label-based similarity scores, we adjust $\mathbf{K}_{j}^{(t+1)}$ by integrating the label-based similarity through $\mathbf{T}_{j}^{(t)}$.

$$
\mathbf{K}_{j}^{(t+1)}=\alpha^{(t+1)} \mathbf{T}_{j}^{(t+1)}+\beta^{(t+1)}\left(\mathbf{T}_{j}^{(t+1)} \mathbf{Y}^{(t)}\right)\left(\mathbf{T}_{j}^{(t+1)} \mathbf{Y}^{(t)}\right)^{T}
$$

subject to $\alpha^{(t+1)}+\beta^{(t+1)}=1, \alpha^{(t+1)}, \beta^{(t+1)} \geq 0$.

$\alpha^{(t+1)}$ and $\beta^{(t+1)}$ are weighting factors to balance two kinds of similarity scores. The label vicinity $\left(\mathbf{T}_{j}^{(t+1)} \mathbf{Y}^{(t)}\right)\left(\mathbf{T}_{j}^{(t+1)} \mathbf{Y}^{(t)}\right)^{T}$ quantitatively measures the extent of similarity between vertices and their neighbors based on the current labeling.

\subsection{Weight Learning}

Classification analysis often utilizes the F1 score, i.e., the harmonic mean of precision and recall, to evaluate the accuracy of testing instances. The objective of multi-label classification of multigraph is to maximize the Macro-F1 score [32], i.e., the unweighted mean of F1 score on classes. To define the Macro-F1 score, we first introduce an indicator function.

$$
\mathcal{I}\left(\hat{y}_{i}^{j}=1\right)= \begin{cases}1, & \hat{y}_{i}^{j}=1, \\ 0, & \hat{y}_{i}^{j}=0 .\end{cases}
$$

where $\mathcal{I}\left(\hat{y}_{i}^{j}=1\right)$ indicates whether the label $c_{j}$ is assigned to an instance vertex $v_{i}$

Definition 3. [Macro-F1] Let $M G=(V, E)$ be a collaboration multigraph, $y_{i}$ be the true label vector of the $i^{t h}$ instance vertices in $V$ and $\hat{y}_{i}$ be the predicted label vector, and the Macro-F1 score is defined below.

$$
\text { Macro-F1 }=\frac{1}{K} \sum_{j=1}^{K} \frac{2 \sum_{i=l+1}^{N_{C G}} \mathcal{I}\left(\hat{y}_{i}^{j}=1\right) y_{i}^{j}}{\sum_{i=l+1}^{N_{C G}} I\left(\hat{y}_{i}^{j}=1\right)+\sum_{i=l+1}^{N_{C G}} y_{i}^{j}}
$$

Assuming $\theta=\max _{i, j}\left\{\mathbf{Y}(i, j): l+1 \leq i \leq N_{C G}, 1 \leq j \leq K\right\}$, we define an s-shape function to approximate the indicator function.

$$
\mathcal{S}(\mathbf{Y}(i, j))=\left\{\begin{array}{cc}
1, & \mathbf{Y}(i, j)=\theta, \\
0, & \mathbf{Y}(i, j)=0, \\
\mathbf{Y}(i, j) / \theta, & \text { otherwise. }
\end{array}\right.
$$


The decision rule determining $\hat{y}_{i}^{j}=1$ if $\mathbf{Y}(i, j)>\theta / 2$, i.e., $\mathcal{S}(\mathbf{Y}(i, j))>0.5$ is represented as follow.

$\mathcal{I}\left(\hat{y}_{i}^{j}=1\right)=\mathcal{I}(\mathbf{Y}(i, j)>\theta / 2)=\mathcal{I}(\mathcal{S}(\mathbf{Y}(i, j))>0.5) \approx \mathcal{S}(\mathbf{Y}(i, j))$

The Macro-F1 score is thus approximated as follow.

$$
\text { Macro-F } \approx \frac{1}{K} \sum_{j=1}^{K} \frac{2 \sum_{i=l+1}^{N_{C G}} \mathbf{Y}(i, j) y_{i}^{j}}{\sum_{i=l+1}^{N_{C G}} \mathbf{Y}(i, j)+\sum_{i=l+1}^{N_{C G}} \theta y_{i}^{j}}
$$

According to Eqs.(3)-(17), the Macro-F1 score is a fractional function of multi variables $\alpha, \beta, \omega_{1}, \cdots, \omega_{N}$ with non-negative real coefficients. On the other hand, the numerator and the denominator of Macro-F1 are both polynomial functions of the above variables. Without loss of generality, we rewrite Eq.(22) as follow.

$$
\begin{gathered}
\text { Macro-F1 } \approx \frac{\sum_{i=1}^{m} a_{i}(\alpha)^{b_{i}}(\beta)^{c_{i}} \prod_{j=1}^{N}\left(\omega_{j}\right)^{d_{i j}}}{\sum_{i=1}^{n} o_{i}(\alpha)^{p_{i}}(\beta)^{q_{i}} \prod_{j=1}^{N}\left(\omega_{j}\right)^{r_{i j}},} \\
a_{i}, b_{i}, c_{i}, d_{i j}, o_{i}, p_{i}, q_{i}, r_{i j} \geq 0, b_{i}, c_{i}, d_{i j}, p_{i}, q_{i}, r_{i j} \in \mathbb{Z}
\end{gathered}
$$

where there are $m$ polynomial terms in the numerator and $n$ polynomial terms in the denominator, $a_{i}$ and $o_{i}$ are the coefficients of the $i^{\text {th }}$ terms respectively, and $b_{i}, c_{i}, d_{i j}, p_{i}, q_{i}, r_{i j}$ are the exponents of corresponding variables in the $i^{\text {th }}$ terms respectively.

Definition 4. [Multigraph Classification Objective] Let $M G=$ $(V, E)$ be a collaboration multigraph, $\alpha, \beta, \omega_{1}, \cdots, \omega_{N}$ are the weighting factors defined in Eqs.(3) and (17), respectively. The goal of multi-label classification of multigraph is to maximize the MacroF1 score.

$$
\max _{\alpha, \beta, \omega_{j}} \text { Macro-F1 } \approx \max _{\alpha, \beta, \omega_{j}} \frac{\sum_{i=1}^{m} a_{i}(\alpha)^{b_{i}}(\beta)^{c_{i}} \prod_{j=1}^{N}\left(\omega_{j}\right)^{d_{i j}}}{\sum_{i=1}^{n} o_{i}(\alpha)^{p_{i}}(\beta)^{q_{i}} \prod_{j=1}^{N}\left(\omega_{j}\right)^{r_{i j}}}
$$

subject to $\alpha+\beta=1, \alpha, \beta \geqslant 0, \sum_{j=1}^{N} \omega_{j}=1, \omega_{j} \geqslant 0, j=1, \cdots, N$.

For ease of presentation, we revise the original objective as the following nonlinear fractional programming problem (NFPP).

Definition 5. [Nonlinear Fractional Programming Problem] Let $f\left(\alpha, \beta, \omega_{1}, \ldots, \omega_{N}\right)=\sum_{i=1}^{m} a_{i}(\alpha)^{b_{i}}(\beta)^{c_{i}} \prod_{j=1}^{N}\left(\omega_{j}\right)^{d_{i j}}$ and $g\left(\alpha, \beta, \omega_{1}, \ldots\right.$ ,$\left.\omega_{N}\right)=\sum_{i=1}^{n} o_{i}(\alpha)^{p_{i}}(\beta)^{q_{i}} \prod_{j=1}^{N}\left(\omega_{j}\right)^{r_{i j}}$, the classification goal is revised below.

$$
\max _{\alpha, \beta, \omega_{1}, \ldots, \omega_{N}} \frac{f\left(\alpha, \beta, \omega_{1}, \ldots, \omega_{N}\right)}{g\left(\alpha, \beta, \omega_{1}, \ldots, \omega_{N}\right)}
$$

subject to $\alpha+\beta=1, \alpha, \beta \geqslant 0, \sum_{i=1}^{N} \omega_{i}=1, \omega_{i} \geqslant 0, i=1, \cdots, N$.

Our classification objective is equivalent to maximize a quotient of two polynomial functions of multiple variables. It is very hard to perform function trend identification and estimation to determine the existence and uniqueness of solutions. Therefore, we want to transform this sophisticated NFPP into a easily solvable problem.

THeorem 1. The NFPP in Definition 5 is equivalent to a polynomial programming problem with polynomial constraints (PPPPC).

$$
\max _{\alpha, \beta, \omega_{1}, \ldots, \omega_{N}, \pi} \pi f\left(\alpha, \beta, \omega_{1}, \ldots, \omega_{N}\right)
$$

subject to $\alpha+\beta=1, \alpha, \beta \geqslant 0, \sum_{i=1}^{N} \omega_{i}=1, \omega_{i} \geqslant 0, i=1, \cdots, N$, $0 \leqslant \pi \leqslant 1 / g\left(\alpha, \beta, \omega_{1}, \ldots, \omega_{N}\right)$.

Proof. If $\left(\bar{\alpha}, \bar{\beta}, \bar{\omega}_{1}, \ldots, \bar{\omega}_{N}, \bar{\pi}\right)$ is an optimal solution of PPP$P C$, then $\bar{\pi}=1 / g\left(\bar{\alpha}, \bar{\beta}, \bar{\omega}_{1}, \ldots, \bar{\omega}_{N}\right)$. Thus $\bar{\pi} f\left(\bar{\alpha}, \bar{\beta}, \bar{\omega}_{1}, \ldots, \bar{\omega}_{N}\right)=$ $f\left(\bar{\alpha}, \bar{\beta}, \bar{\omega}_{1}, \ldots, \bar{\omega}_{N}\right) / g\left(\bar{\alpha}, \bar{\beta}, \bar{\omega}_{1}, \ldots, \bar{\omega}_{N}\right)$. For any feasible solution $\left(\alpha, \beta, \omega_{1}, \ldots, \omega_{N}\right)$ of NFPP, the constraints of PPPPC are satisfied by setting $\pi=1 / g\left(\alpha, \beta, \omega_{1}, \ldots, \omega_{N}\right)$, so $\pi f\left(\alpha, \beta, \omega_{1}, \ldots, \omega_{N}\right) \leqslant$ $\bar{\pi} f\left(\bar{\alpha}, \bar{\beta}, \bar{\omega}_{1}, \ldots, \bar{\omega}_{N}\right)$, i.e. $f\left(\alpha, \beta, \omega_{1}, \ldots, \omega_{N}\right) / g\left(\alpha, \beta, \omega_{1}, \ldots, \omega_{N}\right) \leqslant$ $f\left(\bar{\alpha}, \bar{\beta}, \bar{\omega}_{1}, \ldots, \bar{\omega}_{N}\right) / g\left(\bar{\alpha}, \bar{\beta}, \bar{\omega}_{1}, \ldots, \bar{\omega}_{N}\right)$.

Conversely, if $\left(\bar{\alpha}, \bar{\beta}, \bar{\omega}_{1}, \ldots, \bar{\omega}_{N}\right)$ solves NFPP, then for any feasible solution $\left(\alpha, \beta, \omega_{1}, \ldots, \omega_{N}, \pi\right)$ of PPPPC we have $\pi f\left(\alpha, \beta, \omega_{1}, \ldots\right.$, $\left.\omega_{N}\right) \leqslant f\left(\alpha, \beta, \omega_{1}, \ldots, \omega_{N}\right) / g\left(\alpha, \beta, \omega_{1}, \ldots, \omega_{N}\right) \leqslant f\left(\bar{\alpha}, \bar{\beta}, \bar{\omega}_{1}, \ldots, \bar{\omega}_{N}\right)$ $/ g\left(\bar{\alpha}, \bar{\beta}, \bar{\omega}_{1}, \ldots, \bar{\omega}_{N}\right)=\bar{\pi} f\left(\bar{\alpha}, \bar{\beta}, \bar{\omega}_{1}, \ldots, \bar{\omega}_{N}\right)$ with $\bar{\pi}=1 / g\left(\bar{\alpha}, \bar{\beta}, \bar{\omega}_{1}, \ldots\right.$ , $\left.\bar{\omega}_{N}\right)$.

Although PPPPC is a polynomial programming problem, the polynomial constraints make it very hard to solve. We further simplify it as an nonlinear parametric programming problem (NPPP).
Definition 6. [Nonlinear Parametric Programming Problem] Let $f\left(\alpha, \beta, \omega_{1}, \ldots, \omega_{N}\right)=\sum_{i=1}^{m} a_{i}(\alpha)^{b_{i}}(\beta)^{c_{i}} \prod_{j=1}^{N}\left(\omega_{j}\right)^{d_{i j}}$ and $g\left(\alpha, \beta, \omega_{1}, \ldots\right.$, $\left.\omega_{N}\right)=\sum_{i=1}^{n} o_{i}(\alpha)^{p_{i}}(\beta)^{q_{i}} \prod_{j=1}^{N}\left(\omega_{j}\right)^{r_{i j}}$, the NPPP is defined below.

$F(\gamma)=\max _{\alpha, \beta, \omega_{1}, \ldots, \omega_{N}} f\left(\alpha, \beta, \omega_{1}, \ldots, \omega_{N}\right)-\gamma g\left(\alpha, \beta, \omega_{1}, \ldots, \omega_{N}\right)$

subject to $\alpha+\beta=1, \alpha, \beta \geqslant 0, \sum_{i=1}^{N} \omega_{i}=1, \omega_{i} \geqslant 0, i=1, \cdots, N$.

Theorem 2. The NFPP in Definition 5 is equivalent to the NPP$P$ in Definition 6, i.e.,

$\gamma=\max _{\alpha, \beta, \omega_{1}, \ldots, \omega_{N}} \frac{f\left(\alpha, \beta, \omega_{1}, \ldots, \omega_{N}\right)}{g\left(\alpha, \beta, \omega_{1}, \ldots, \omega_{N}\right)}$ iff $F(\gamma)=\max _{\alpha, \beta, \omega_{1}, \ldots, \omega_{N}} f\left(\alpha, \beta, \omega_{1}, \ldots, \omega_{N}\right)-$ $\gamma g\left(\alpha, \beta, \omega_{1}, \ldots, \omega_{N}\right)=0$.

Proof. If $\left(\bar{\alpha}, \bar{\beta}, \bar{\omega}_{1}, \ldots, \bar{\omega}_{N}\right)$ is a feasible solution of $F(\gamma)=0$, then $f\left(\bar{\alpha}, \bar{\beta}, \bar{\omega}_{1}, \ldots, \bar{\omega}_{N}\right)-\gamma g\left(\bar{\alpha}, \bar{\beta}, \bar{\omega}_{1}, \ldots, \bar{\omega}_{N}\right)=0$. Thus $f\left(\alpha, \omega_{1}, \ldots\right.$ ,$\left.\omega_{N}\right)-\gamma g\left(\alpha, \omega_{1}, \ldots, \omega_{N}\right) \leqslant f\left(\bar{\alpha}, \bar{\beta}, \bar{\omega}_{1}, \ldots, \bar{\omega}_{N}\right)-\gamma g\left(\bar{\alpha}, \bar{\beta}, \bar{\omega}_{1}, \ldots, \bar{\omega}_{N}\right)$ $=0$. We have $\gamma=f\left(\bar{\alpha}, \bar{\beta}, \bar{\omega}_{1}, \ldots, \bar{\omega}_{N}\right) / g\left(\bar{\alpha}, \bar{\beta}, \bar{\omega}_{1}, \ldots, \bar{\omega}_{N}\right) \geqslant f\left(\alpha, \omega_{1}\right.$, $\left.\ldots, \omega_{N}\right) / g\left(\alpha, \omega_{1}, \ldots, \omega_{N}\right)$. Therefore, $\gamma$ is a maximum value of $N F$ $P P$ and $\left(\bar{\alpha}, \bar{\beta}, \bar{\omega}_{1}, \ldots, \bar{\omega}_{N}\right)$ is an optimal solution of NFPP.

Conversely, if $\left(\bar{\alpha}, \bar{\beta}, \bar{\omega}_{1}, \ldots, \bar{\omega}_{N}\right)$ solves NFPP, then we have $\gamma=$ $f\left(\bar{\alpha}, \bar{\beta}, \bar{\omega}_{1}, \ldots, \bar{\omega}_{N}\right) / g\left(\bar{\alpha}, \bar{\beta}, \bar{\omega}_{1}, \ldots, \bar{\omega}_{N}\right) \geqslant f\left(\alpha, \omega_{1}, \ldots, \omega_{N}\right) / g\left(\alpha, \omega_{1}\right.$, $\left.\ldots, \omega_{N}\right)$. Thus $f\left(\alpha, \omega_{1}, \ldots, \omega_{N}\right)-\gamma g\left(\alpha, \omega_{1}, \ldots, \omega_{N}\right) \leqslant f\left(\bar{\alpha}, \bar{\beta}, \bar{\omega}_{1}, \ldots\right.$ , $\left.\bar{\omega}_{N}\right)-\gamma g\left(\bar{\alpha}, \bar{\beta}, \bar{\omega}_{1}, \ldots, \bar{\omega}_{N}\right)=0$. We have $F(\gamma)=0$ and the maximum is taken at $\left(\bar{\alpha}, \bar{\beta}, \bar{\omega}_{1}, \ldots, \bar{\omega}_{N}\right)$.

Now the original NFPP has been successfully transformed into the straightforward NPPP. This transformation can efficiently speed up the classification convergence due to the following properties.

Theorem 3. $F(\gamma)$ is convex.

Proof: Suppose that $\left(\bar{\alpha}, \bar{\beta}, \bar{\omega}_{1}, \ldots, \bar{\omega}_{N}\right)$ is an optimal solution of $F\left((1-\lambda) \gamma_{1} \pm \lambda \gamma_{2}\right)$ with $\gamma_{1} \neq \gamma_{2}$ and $0 \leqslant \lambda \leqslant 1 . F\left((1-\lambda) \gamma_{1}+\right.$ $\left.\lambda \gamma_{2}\right)=\underline{f}\left(\bar{\alpha}, \bar{\beta}, \bar{\omega}_{1}, \ldots, \bar{\omega}_{N}\right)-\left((1-\lambda) \gamma_{1}+\lambda \gamma_{2}\right) g\left(\bar{\alpha}, \bar{\beta}, \bar{\omega}_{1}, \ldots, \bar{\omega}_{N}\right)=$ $\lambda\left(f\left(\bar{\alpha}, \bar{\beta}, \bar{\omega}_{1}, \ldots, \bar{\omega}_{N}\right)-\gamma_{2} g\left(\bar{\alpha}, \bar{\beta}, \bar{\omega}_{1}, \ldots, \bar{\omega}_{N}\right)\right)+(1-\lambda)\left(f\left(\bar{\alpha}, \bar{\beta}, \bar{\omega}_{1}, \ldots\right.\right.$, $\left.\left.\bar{\omega}_{N}\right)-\gamma_{1} g\left(\bar{\alpha}, \bar{\beta}, \bar{\omega}_{1}, \ldots, \bar{\omega}_{N}\right)\right) \leqslant \lambda \max _{\alpha, \beta, \omega_{1}, \ldots, \omega_{N}} f\left(\alpha, \beta, \omega_{1}, \ldots, \omega_{N}\right)-\gamma_{2} g(\alpha$, $\left.\beta, \omega_{1}, \ldots, \omega_{N}\right)+(1-\lambda) \max _{\alpha, \beta, \omega_{1}, \ldots, \omega_{N}} f\left(\alpha, \beta, \omega_{1}, \ldots, \omega_{N}\right)-\gamma_{1} g\left(\alpha, \beta, \omega_{1}, \ldots\right.$, $\left.\omega_{N}\right)=\lambda F\left(\gamma_{2}\right)+(1-\lambda) F\left(\gamma_{1}\right)$. Thus, $F(\gamma)$ is convex.

Theorem 4. $F(\gamma)$ is monotonically decreasing.

Proof: Suppose that $\gamma_{1}>\gamma_{2}$ and $\left(\bar{\alpha}, \bar{\beta}, \bar{\omega}_{1}, \ldots, \bar{\omega}_{N}\right)$ is an optimal solution of $F\left(\gamma_{1}\right)$. Thus, $F\left(\gamma_{1}\right)=f\left(\bar{\alpha}, \bar{\beta}, \bar{\omega}_{1}, \ldots, \bar{\omega}_{N}\right)-\gamma_{1} g\left(\bar{\alpha}, \bar{\beta}, \bar{\omega}_{1}, \ldots\right.$ , $\left.\bar{\omega}_{N}\right)<f\left(\bar{\alpha}, \bar{\beta}, \bar{\omega}_{1}, \ldots, \bar{\omega}_{N}\right)-\gamma_{2} g\left(\bar{\alpha}, \bar{\beta}, \bar{\omega}_{1}, \ldots, \bar{\omega}_{N}\right) \leqslant \max _{\alpha, \beta, \omega_{1}, \ldots, \omega_{N}} f(\alpha$, $\left.\beta, \omega_{1}, \ldots, \omega_{N}\right)-\gamma_{2} g\left(\alpha, \beta, \omega_{1}, \ldots, \omega_{N}\right)=F\left(\gamma_{2}\right)$.

Theorem 5. $F(\gamma)=0$ has a unique solution.

Proof: Based on the above-mentioned theorems, we know $F(\gamma)$ is continuous as well as decreasing. In addition, $\lim _{\gamma \rightarrow+\infty} F(\gamma)=-\infty$ and $\lim _{\gamma \rightarrow-\infty} F(\gamma)=+\infty$.

The procedure of solving this NPPP includes two parts: (1) find such a reasonable parameter $\gamma(F(\gamma)=0)$, making NPPP equivalent to NFPP; (2) given the parameter $\gamma$, solve a polynomial programming problem about the original variables $\alpha, \beta, \omega_{1}, \ldots, \omega_{N}$. Our weight adjustment mechanism is an iterative procedure to find the solution of $F(\gamma)=0$ and the corresponding weights after each iteration of the classification process. We first generate an initial unified classification kernel $\mathbf{K}_{j}^{(1)}$ with equal weights of $\frac{1}{N}$ to produce an initial classification result on the collaboration multigraph. According to the initial classification result, we then calculate an initial $F(\gamma)$. Since $F(\gamma)$ is a monotonic decreasing function and $F(0)=\max _{\alpha, \beta, \omega_{1}, \ldots, \omega_{N}} f\left(\alpha, \beta, \omega_{1}, \ldots, \omega_{N}\right)$ is obviously non-negative, we start with an initial $\gamma=0$ and solve the subproblem $F(0)$ by using existing fast polynomial programming model to update the weights $\alpha, \beta, \omega_{1}, \ldots, \omega_{N}$. The parameter $\gamma$ is gradually increased by $\gamma=f\left(\alpha, \beta, \omega_{1}, \ldots, \omega_{N}\right) / g\left(\alpha, \beta, \omega_{1}, \ldots, \omega_{N}\right)$ to help the algorith$\mathrm{m}$ enter the next round. The algorithm repeats the above-mentioned iterative procedure until $F(\gamma)$ converges to 0 .

By assembling different pieces together, we provide the pseudo code of our AEClass classifier in Algorithm 1. 


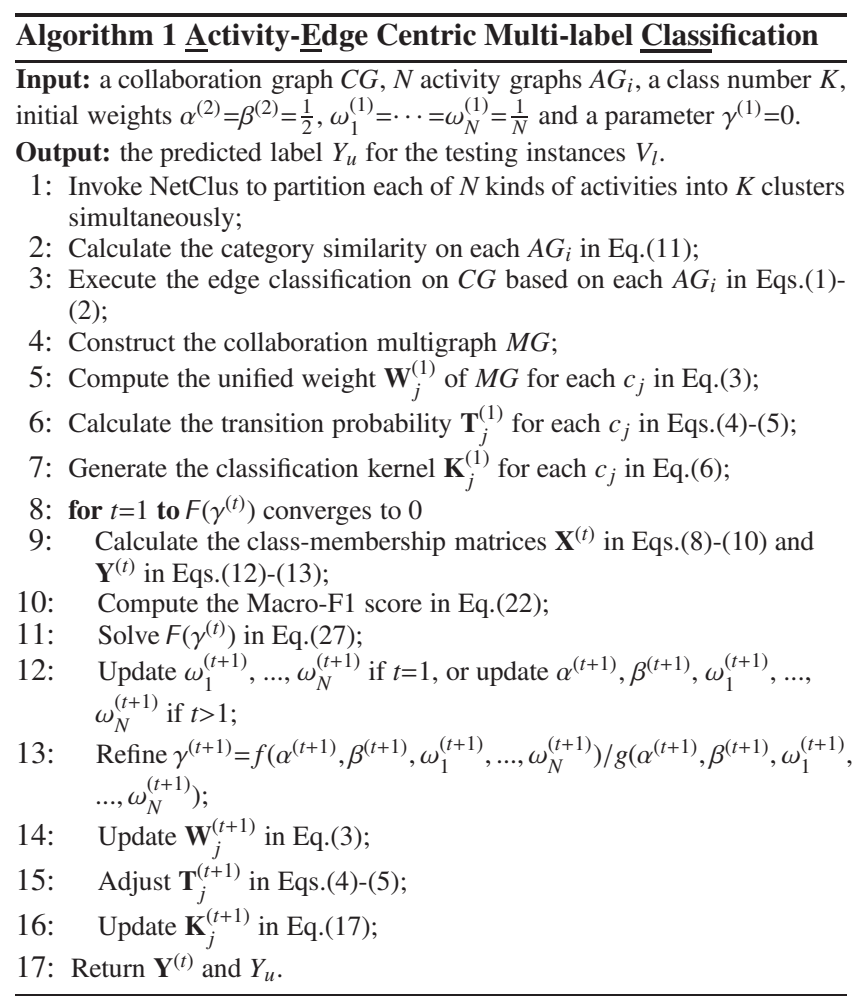

\section{EXPERIMENTAL EVALUATION}

We have performed extensive experiments to evaluate the performance of our AEClass classifier on real graph datasets.

\subsection{Experimental Datasets}

The first real-world dataset is extracted from the DBLP Bibliography data ${ }^{1}$. We build a coauthor graph with highly prolific 100,000 authors from all research areas and 712, 834 associated links where vertices represent authors and edges represent their coauthor relationships, and two associated activity graphs: conference graph and term graph. According to [31], we categorize research areas into 24 fields: AI, AIGO, ARC, BIO, CV, DB, DIST, DM, EDU, GRP, HCI, IR, ML, MUL, NLP, NW, OS, PL, RT, SC, SE, SEC, SIM, WWW. We utilize a multi-typed soft clustering framework, NetClus [25], to cluster conferences and terms into 24 categories simultaneously. According to conference's or term's clustering distribution over 24 categories and ranking score in each category, we calculate the similarities between conferences or terms. The classification goal is infer research areas of each author.

Last.fm ${ }^{2}$ is a music-oriented online social network. We use the API call user.getfriends to collect the list of friends and construct a friendship graph with 50,000 users and 496,611 associated links where vertices represent users and edges denote their friendships. The two activity networks: artist graph and track graph are generated by invoking the API calls artist.getSimilar and track.getSimilar respectively. By calling the API calls user.getTopArtists and user.getTopTracks, we classify each friendship edge in terms of the same artists or the same tracks shared by two users. The classification task is to assign each user to a subset of 21 music genres in the database: acoustic, ambient, blues, classical, country, electronic, emo, folk, hardcore, hip hop, indie, jazz, latin, metal, pop, pop punk, punk, reggae, rnb, rock, soul.

\footnotetext{
${ }^{1}$ http://dblp.uni-trier.de/xml/

${ }^{2}$ http://www.last.fm/api
}

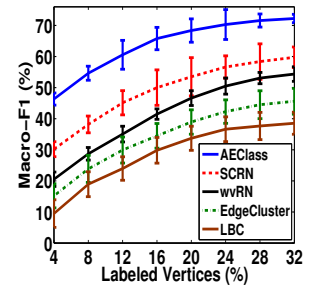

(a) Macro-F1

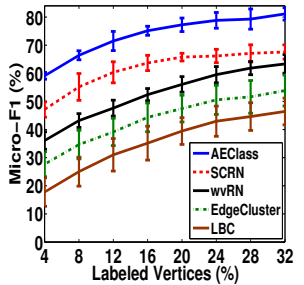

(b) Micro-F1

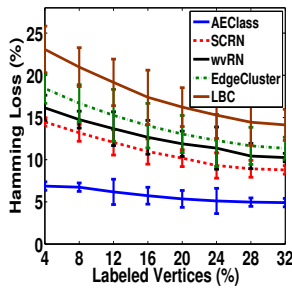

(c) Hamming Loss
Figure 7: Classification Quality on DBLP

The third real dataset is extracted from the Internet Movie Database $(\mathrm{IMDb})^{3}$. We construct a collaboration graph with 10,000 highly prolific actors and 270, 227 links where vertices represent actors and edges specify their costar relationships in terms of coappearance of actors in the same movies. We build a movie activity graph where edges denote co-direct relationship between movies, i.e., movies are directed by the same directors. The objective is to associate each actor with a subset of 22 movie genres: Action, Adventure, Animation, Biography, Comedy, Crime, Documentary, Drama, Family, Fantasy, Film-Noir, History, Horror, Music, Musical, Mystery, Romance, Sci-Fi, Sport, Thriller, War, Western.

\subsection{Comparison Methods and Evaluation}

We compare AEClass with two representative link-based classification algorithms, LBC [1], wvRN [2], and two recently developed multi-label classifiers, EdgeCluster [18], SCRN [20]. All four methods perform multi-label classification on a single weighted graph based on the assumption of vertex homophily. The detailed introductions for four methods are presented in Section 5. Note that LBC is originally a multi-class classifier. In order to compare all algorithms, we modify the last step in LBC and use the posterior probability distribution over $K$ classes as the multilabel classification result. AEClass integrates multiple information networks into a unified multigraph with combining both the vertexcentric multi-label classification and the edge-centric multi-label classification based on vertex-edge homophily. It also integrates both the structure affinity and the label vicinity into a unified classifier through dynamic weight tuning mechanism.

Evaluation Measures We use three measures to evaluate the quality of classification results generated by different methods. The first measure is Macro-F1 defined in Definition 3. Given the same definitions in Eq.(18), other two metrics are defined as follows.

$$
\text { Micro-F1 }=\frac{2 \sum_{j=1}^{K} \sum_{i=l+1}^{N_{C G}} \mathcal{I}\left(\hat{y}_{i}^{j}=1\right) y_{i}^{j}}{\sum_{j=1}^{K} \sum_{i=l+1}^{N_{C G}} \mathcal{I}\left(\hat{y}_{i}^{j}=1\right)+\sum_{j=1}^{K} \sum_{i=l+1}^{N_{C G}} y_{i}^{j}}
$$

Micro-F1 [32] represents the harmonic mean of micro average of precision and recall. The larger the value, the better the quality.

$$
\text { HammingLoss }=\frac{1}{N_{C G}-l} \sum_{i=l+1}^{N_{C G}} \frac{1}{K}\left\|\mathcal{I}\left(\hat{y}_{i}=1\right) \oplus y_{i}\right\|_{1}
$$

where $\oplus$ represents the XOR operation, and $\|\cdot\|_{1}$ specifies the $l 1$ norm. Hamming Loss [33] measures the loss between true labels and predicted labels. The smaller the value, the better the quality.

\subsection{Classification Quality}

Figures 7-9 exhibit the classification quality on DBLP, Last.fm and IMDb by varying the proportion of labeled vertices respectively. For each proportion of labeled vertices, we average the performance scores over 10 cross-validation folds. The average performance scores with standard deviations of five multi-label classification methods are reported with respect to three evaluation measures of Macro-F1, Micro-F1 and Hamming Loss. We make the following observations on the performances by different methods.

\footnotetext{
${ }^{3}$ http://www.imdb.com/interfaces
} 


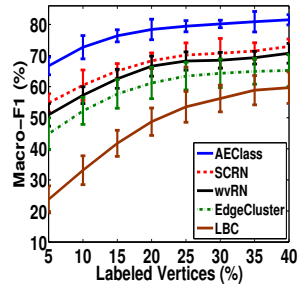

(a) Macro-F1

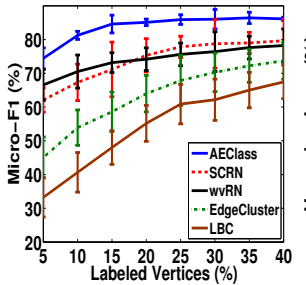

(b) Micro-F1

Figure 8: Classification Quality on Last.fm

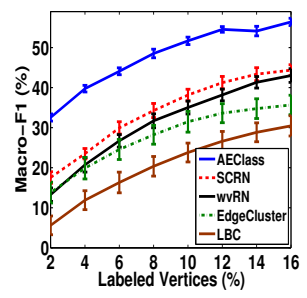

(a) Macro-F1

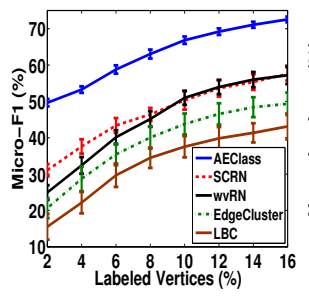

(b) Micro-F1

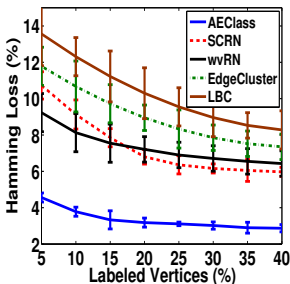

(c) Hamming Loss

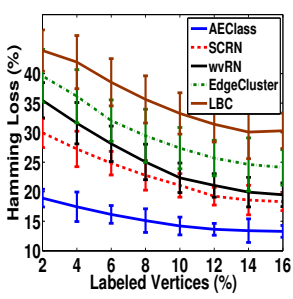

(c) Hamming Loss

Figure 9: Classification Quality on IMDb

First, AEClass, SCRN and wvRN significantly outperform LBC and EdgeCluster on all three evaluation measures. We first categorize five multi-label classification methods into non-transductive learning methods and transductive learning methods, based on how they utilize topological structure information. As non-transductive learning methods, both LBC and EdgeCluster only utilize the direct links between vertices in the graph, i.e., one-hop structure information, to produce vertex's features. As transductive learning approaches, AEClass, SCRN and wvRN make full use of both direct links and indirect edges (the circle of friends) between vertices through iterative graph propagation, i.e., multiple-hop structure information, to further improve the classification quality. These results demonstrate the importance of exploiting both direct links and indirect edges for multi-label classification in networked data.

Second, SCRN always outperforms wvRN on three graph datasets. Although SCRN and wvRN exploit the very similar relational inference framework, SCRN improves wvRN by integrating both the network topology and the social context features extracted by EdgeCluster into the classifier. A careful examination reveals that these two approaches are very close in terms of prediction performance in many situations, in spite of the optimization adopted by SCRN. A reasonable explanation is that both of them are only based on the assumption of vertex homophily, i.e., the principle that similar vertices in nature are connected to each other with social links.

Finally, among all five classification methods, AEClass achieves the best classification performance on all three real datasets for al1 three evaluation measures. Compared to other algorithms, AEClass averagely achieves $14.6 \%$ Macro-F1 increase, $12.1 \%$ MicroF1 boost and 5.2\% loss reduction on DBLP, 10.2\% Macro-F1 growth, 9.9\% Micro-F1 increase and $4.1 \%$ loss decrease on Last.fm, and $16.7 \%$ Macro-F1 increase, $16.2 \%$ Micro-F1 boost and $7.5 \%$ loss reduction on IMDb, respectively. Note that even if the proportion of labeled vertices is very small, such as $2 \%$ and $4 \%$, AEClass still can achieve comparable accuracy on all datasets. Concretely, there are four critical reasons for high accuracy of AEClass: (1) the structure information from associated activity networks boosts the effectiveness of classification. Activity network partition provides us with additional activity labels; (2) the multigraph organization integrates both the vertex-centric multi-label classification based on vertex homophily and the edge-centric multi-label classification based on vertex-edge homophily to leverage the classification performance; (3) Activity network partition captures the inter-dependencies among multiple class labels; and (4) the iterative learning algorithm help the classifier achieve a good balance among different activity-

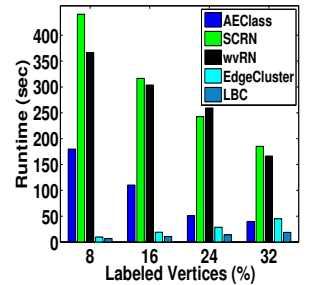

(a) DBLP

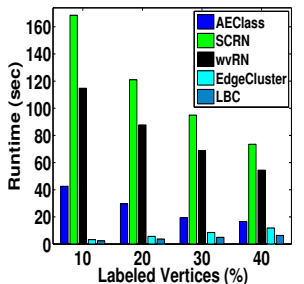

(b) Last.fm

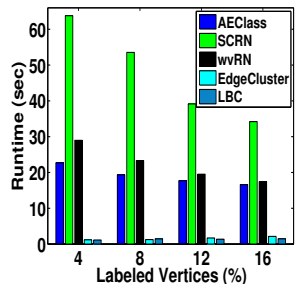

(c) IMDb
Figure 10: Classification Efficiency

based edge classification schemes and an effective integration of the structure affinity and the label vicinity.

\subsection{Classification Efficiency}

Figures 10 (a), (b) and (c) present the classification time on DBLP, Last.fm and IMDb with different proportions of labeled vertices respectively. First, LBC has lowest runtime in seconds compared to all other algorithms in all experiments, since it is a logistic regression classifier by aggregating the labels of neighbors as vertex's feature vector. Second, EdgeCluster, a linear SVM classifier with an edge clustering scheme to extract sparse social dimension$\mathrm{s}$, is slightly slower than LBC since the linear SVM approaches generally fall behind the LR methods in speed. Both LBC and EdgeCluster are faster than other three methods because both only utilize the direct links between vertices, i.e., one-hop structure information. In comparison, AEClass, SCRN and wvRN use both direct links and indirect edges (the circle of friends) between vertices, i.e., multiple-hop structure information. Thus, the last three classifiers have higher time complexity than the first two models but they achieve better classification quality. Third, wvRN is consistently faster than SCRN on all three datasets. SCRN improves wvRN by integrating the social dimensions extracted by EdgeCluster into the classifier. This improvement results in an additional computational cost for calculating the class propagation probability of each vertex on each class. Finally, AEClass significantly outperforms the other two transductive learning based multi-label classifiers: SCRN and wvRN. Although SCRN and wvRN execute the classification on a general graph, AEClass does classification on an activity-based collaboration multigraph by augmenting its edges with class labels from each activity graph. There are three main reasons for high efficiency of AEClass: (1) the multigraph organization increases the size of dataset but reduces the computational cost of classification. As we discussed in Subsection 3.2, based on the vertex homophily, no matter which class the current objective is, both SCRN and wvRN need to check each neighbor of a vertex and summarize the labels of all neighbors. In contrast, AEClass only picks up those vertices and links with the same label as the current objective class to execute the inference. Most importantly, AEClass stops the label propagation through irrelevant neighbors (without a link with the same label as the current objective class) in the future iterations; (2) the label vicinity between vertices based on the class-membership distribution over $K$ classes is integrated into the classifier; and (3) we transform the original nonlinear fractional programming problem of multiple weights into a nonlinear parametric programming problem of single variable. According to Theorems 2-5, solving $F(\gamma)$ for a given $\gamma$ is a polynomial programming problem which can be sped up by existing fast polynomial programming model.

\subsection{Classification Convergence}

Figure 11 (a) and (b) exhibit the trend of classification convergence in terms of Macro-F1, Micro-F1, and Hamming Loss on DBLP with 4\% label nodes and Last.fm with 5\% label vertices. Both the Macro-F1 values and the Micro-F1 scores in two figures keep increasing and have concave curves when we iteratively perform the tasks of vertex labeling, weight update and kernel adjustment 


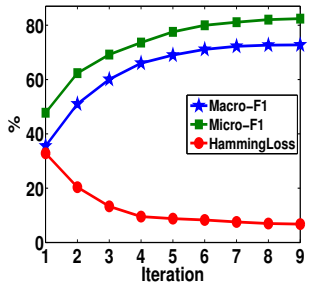

(a) DBLP

Figure 11: Classification Convergence

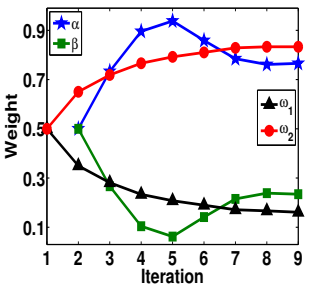

(a) DBLP

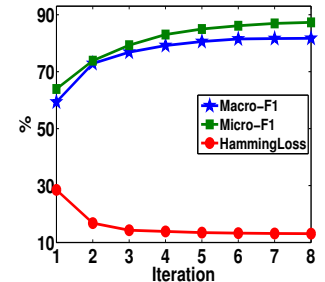

(b) Last.fm

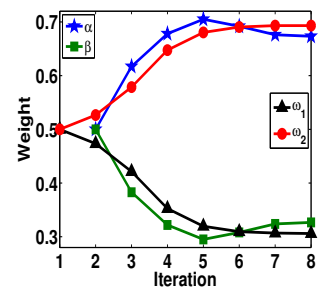

(b) Last.fm
Figure 12: Weight Update

during the classification process. On the other hand, the Hamming Loss values decrease with the classification iterations and have a convex curve. The classification process converges very quickly, usually in eight iterations for Last.fm and nine iterations for DBLP.

Figure 12 (a) and (b) show the tendency of weight update on DBLP and Last.fm respectively. $\alpha$ and $\beta$ in two figures represen$\mathrm{t}$ the weights of structure affinity and label vicinity in the unified classifier $\mathbf{K}_{j}^{(t+1)}$ in Eq.(17) respectively. $\omega_{1}$ and $\omega_{2}$ in Figure 12 (a) denote the weights of the conference graph and the term graph respectively. $\omega_{1}$ and $\omega_{2}$ in Figure 12 (b) represent the weights of the artist graph and the track graph respectively. We keep the constraints $\alpha+\beta=1$ and $\omega_{1}+\omega_{2}=1$ during the classification process. We observe that all the weights converge as the clustering process converges. An interesting phenomenon is that $\alpha$ first increases and then decreases with the iterations and the $\beta$ curve has a converse trend. A reasonable explanation is that there is lack of enough labeling information at the beginning of classification such that the unified classifier has to rely mostly on the structure affinity to achieve a good classification performance. After a few iterations, we have enough labeling information to utilize both the structure affinity and the label vicinity to classify vertices. An interesting finding is that the term weight is increasing but the conference weight is decreasing with more iterations. A reasonable explanation is that people who have many publications on the same conferences may have different research topics but people who have many papers with the same terms usually have the same research topics. For example, both database papers and data mining papers are published on $V L D B$. Similarly, the track weight increases but the artist weight decreases with more iterations. This is because users who favor the same artists may belong to different music genres since the artist$\mathrm{s}$ are often related to multiple genres but users who like the same tracks usually belong to the same music genres.

\subsection{Case Study}

We examine some details of the experiment results on DBLP 100,000 Authors when the proportion of labeled vertices is equal to $32 \%$ based on the coauthor graph, the conference graph and the term graph. Table 1 shows the set of authors and their classmembership probabilities after seven iterations based on 24 conference categories and 24 term categories. We only present most prolific DBLP experts in the area of database (DB), data mining (DM), machine learning (ML) and information retrieval (IR). The class-membership scores in Table 1 are normalized by differen$t$ (conference or term) categories for each author. We observe that the predicted class memberships of authors are consistent with their

\begin{tabular}{|c|c|c|c|c|}
\hline Author/Class & DB & DM & ML & IR \\
\hline Peter L. Bartlett & 0.019 & 0.039 & 0.938 & 0.004 \\
\hline Elisa Bertino & 0.738 & 0.054 & 0.028 & 0.180 \\
\hline Andrei Z. Broder & 0.037 & 0.097 & 0.015 & 0.851 \\
\hline Michael J. Carey & 0.965 & 0.029 & 0.006 & 0.023 \\
\hline W. Bruce Croft & 0.054 & 0.007 & 0.037 & 0.902 \\
\hline David J. DeWitt & 0.912 & 0.057 & 0.004 & 0.027 \\
\hline Inderjit S. Dhillon & 0.030 & 0.409 & 0.457 & 0.104 \\
\hline Christos Faloutsos & 0.321 & 0.500 & 0.031 & 0.147 \\
\hline Jiawei Han & 0.391 & 0.463 & 0.045 & 0.100 \\
\hline H. V. Jagadish & 0.850 & 0.056 & 0.009 & 0.048 \\
\hline Michael I. Jordan & 0.007 & 0.062 & 0.917 & 0.014 \\
\hline Daphne Koller & 0.026 & 0.045 & 0.915 & 0.013 \\
\hline Vipin Kumar & 0.120 & 0.622 & 0.199 & 0.059 \\
\hline Bing Liu & 0.086 & 0.427 & 0.266 & 0.220 \\
\hline Hector Garcia-Molina & 0.788 & 0.010 & 0.016 & 0.186 \\
\hline C. J. van Rijsbergen & 0.003 & 0.051 & 0.024 & 0.922 \\
\hline Michael Stonebraker & 0.946 & 0.013 & 0.007 & 0.034 \\
\hline Jeffrey D. Ullman & 0.824 & 0.065 & 0.064 & 0.047 \\
\hline Philip S. Yu & 0.342 & 0.496 & 0.044 & 0.118 \\
\hline Mohammed J. Zaki & 0.148 & 0.672 & 0.057 & 0.123 \\
\hline
\end{tabular}

Table 1: Class-membership Probabilities of Authors Based on Conference and Keyword Partitions from DBLP

actual research areas. For those experts with unique research areas, such as Michael J. Carey and Michael Stonebraker, the primary research areas for them in the predicted result are obviously consistent with their actual research areas; For those researchers known to work in multiple research areas, the predicted class-membership distributions also correspond to their current research activities. For example, both Jiawei Han and Philip S. Yu are experts on data mining and database, though their $D M$ probabilities are slightly higher since each of them and their circle of co-authors have more $D M$ papers. This table also shows that each author has a class-membership score in each category. This demonstrates that our AEClass model can make each author quickly reach each class label.

\section{RELATED WORK}

Node classification in networked data has attracted active research in the last decade [1-9]. LBC [1] is a network-only derivative of the link-based classifier which creates a feature vector for a node by aggregating the labels of neighboring nodes, and then uses logistic regression to build a discriminative model based on these feature vectors. wvRN [2] presented a weighted-vote relational neighbor classifier to solve link-based classification problems based solely on the class labels of linked neighbors. DYCOS [6] exhibited a node classification model in dynamic information networks with both text content and links. RankClass [7] integrates classification and ranking in a mutually enhancing process to provide class summaries for heterogeneous information networks.

Multi-label classification is gaining attention in recent years [1016]. Read et al. [12] reduces the complexity and potential for error with a pruning procedure to focus on core relationships within multi-label sets. IBLR [13] proposed a multi-label classification approach to combine model-based and similarity-based inference with the estimation of optimal regression coefficients. LEAD [15] decomposes a multi-label learning task into a set of single-label classification problems with a Bayesian network to encode the conditional dependencies of labels as well as the feature set. Guo and $\mathrm{Gu}$ [16] proposed a generalized conditional dependency network for model training using binary classifiers and label predictions using Gibbs sampling inference.

Multi-label classification in networked data has been extensively studied in recent years [17-22]. Sun et al. [17] presented a hypergraph spectral learning formulation for multi-label classification, where a hypergraph is constructed to exploit the correlation information among different labels. EdgeCluster [18] presented a socialdimension based approach for collective behavior prediction with 
an edge clustering scheme to extract sparse social dimensions and a linear SVM classifier for discriminative learning. SCRN [20] is a multi-label iterative relational neighbor classifier by considering both network topology and social context features. PIPL [21] facilitates the multi-label learning process by mining label correlations and instance correlations from the heterogeneous networks.

Recent works on heterogeneous social network analysis [7,9,2330] combine links and content into heterogeneous information networks to improve the quality of querying, ranking and clustering. Cai et al. [23] proposed to learn an optimal linear combination of different relations on heterogeneous social networks in terms of their importance on a certain query. GenClus [28] proposed a modelbased method for clustering heterogeneous networks with different link types and different attribute types. Yu et al. [29] presented a query-driven discovery system for finding semantically similar substructures in heterogeneous networks.

To our knowledge, this work is the first one to address the problem of activity-edge centric multi-label classification of heterogeneous multigraph with the prior knowledge of multiple activity graphs by dynamically adjusting their individual contributions.

\section{CONCLUSIONS}

We have presented an edge-centric multi-label classification approach for mining heterogeneous information networks. First, we integrate the primary social network and multiple associated activity networks into a unified multigraph with edge classification. Second, we combine both the structure affinity and the label vicinity based on multiple activity networks into a unified classifier. Third, an iterative learning algorithm is proposed dynamically refine the classification result by continuously adjusting the weights on different activity-based edge classification schemes from multiple activity graphs, while constantly learning the contributions of the structure affinity and the label vicinity in the unified classifier.

Acknowledgement. This material is based upon work partially supported by the NSF under Grants IIS-0905493, CNS-1115375, IIP-1230740, and a grant from Intel ISTC on Cloud Computing.

\section{REFERENCES}

[1] Q. Lu and L. Getoor. Link-based classification. In ICML'03.

[2] S. A. Macskassy and F. Provost. A simple relational classifier. In MRDM, pages 64-76, 2003.

[3] D. Zhou, O. Bousquet, T. N. Lal, J. Weston, and B. Scholkopf. Learning with local and global consistency. In NIPS, 2003.

[4] J. Neville and D. Jensen. Relational dependency networks. Journal of Machine Learning Research, 8:653-692, 2007.

[5] S. A. Macskassy and F. Provost. Classification in networked data: A toolkit and a univariate case study. Journal of Machine Learning Research, 8:935-983, 2007.

[6] C. C. Aggarwal and N. Li. On Node Classification in Dynamic Content-based Networks. In SDM, 2011.

[7] M. Ji, J. Han, and M. Danilevsky. Ranking-based classification of heterogeneous information networks. In KDD, pages 1298-1306, 2011.

[8] S. Bhagat, G. Cormode, and S. Muthukrishnan. Node classification in social networks. Social Network Data Analytics, pages 115-148, 2011.

[9] X. Kong, P. S. Yu, Y. Ding, and D. J. Wild. Meta path-based collective classification in heterogeneous information networks. In CIKM, pages 1567-1571, 2012.

[10] S. Godbole and S. Sarawagi. Discriminative methods for multi-labeled classification. In PAKDD, 2004.
[11] G. Chen, Y. Song, F. Wang, and C. Zhang. Semi-supervised multi-label learning by solving a sylvester equation. In $S D M$, Atlanta, GA, April 24-26 2008.

[12] J. Read, B. Pfahringer, and G. Holmes. Multi-label classification using ensembles of pruned sets. In ICDM'08.

[13] W. Cheng and E. Hullermeier. Combining instance-based learning and logistic regression for multilabel classification. Machine Learning, 76(2-3):211-225, 2009.

[14] K. Dembczynski, W. Cheng, and E. Hullermeier. Bayes optimal multilabel classification via probabilistic classifier chains. In ICML, Haifa, Israel, June 21-24 2010.

[15] M.-L. Zhang and K. Zhang. Multi-label learning by exploiting label dependency. In KDD, 999-1008, 2010.

[16] Y. Guo and S. Gu. Multi-label classification using conditional dependency networks. In IJCAI, 2011.

[17] L. Sun, S. Ji, and J. Ye. Hypergraph spectral learning for multi-label classification. In KDD, 668-676, 2008.

[18] L. Tang and H. Liu. Scalable learning of collective behavior based on sparse social dimensions. In CIKM, 2009.

[19] S. Peters, Y. Jacob, L. Denoyer, and P. Gallinari. Iterative multi-label multi-relational classification algorithm for complex social networks. Social Network Analysis and Mining, 2(1):17-29, 2012.

[20] X. Wang and G. Sukthankar. Multi-label relational neighbor classification using social context features. In KDD, 2013.

[21] X. Kong, B. Cao, and P. S. Yu. Multi-label classification by mining label and instance correlations from heterogeneous information networks. In KDD, pages 614-622, 2013.

[22] B. Wang, Z. Tu, and J. K. Tsotsos. Dynamic label propagation for semi-supervised multi-class multi-label classification. In ICCV, 2013.

[23] D. Cai, Z. Shao, X. He, X. Yan, and J. Han. Community mining from multi-relational networks. In PKDD, 2005.

[24] Y. Zhou, H. Cheng, and J. X. Yu. Graph clustering based on structural/attribute similarities. In VLDB, 718-729, 2009.

[25] Y. Sun, Y. Yu, and J. Han. Ranking-based clustering of heterogeneous information networks with star network schema. In KDD, pages 797-806, 2009.

[26] T. Yang, R. Jin, Y. Chi, and S. Zhu. Combining link and content for community detection: a discriminative approach. In $K D D$, pages 927-936, 2009.

[27] Y. Zhou, H. Cheng, and J. X. Yu. Clustering large attributed graphs: An efficient incremental approach. In ICDM, 2010.

[28] Y. Sun, C. C. Aggarwal, and J. Han. Relation strength-aware clustering of heterogeneous information networks with incomplete attributes. PVLDB, 5(5):394-405, 2012.

[29] X. Yu, Y. Sun, P. Zhao, and J. Han. Query-driven discovery of semantically similar substructures in heterogeneous networks. In KDD, 1500-1503, 2012.

[30] Y. Zhou and L. Liu. Social influence based clustering of heterogeneous information networks. In KDD, 2013.

[31] T. Chakraborty, S. Sikdar, V. Tammana, N. Ganguly, and A. Mukherjee. Computer science fields as ground-truth communities: Their impact, rise and fall. In ASONAM, 2013.

[32] R.-E. Fan and C.-J. Lin. A study on threshold selection for multi-label classification. In Tech Report, National Taiwan University, 2007.

[33] X. Zhang, Q. Yuan, S. Zhao, W. Fan, W. Zheng, and Z. Wang. Multi-label classification without the multi-label cost. In SDM, 2010. 